\title{
The Development of Social Representations of Gender
}

\author{
GERARD DUVEEN \\ University of Cambridge, United Kingdom
}

\begin{abstract}
Some aspects of the ontogenesis of social representations are discussed through a consideration of specific issues raised by empirical research on the development of representations of gender through children's first year of schooling. A comparison with Vygotsky's general law of cultural development emphasises the importance of considering social identities as structures mediating between the interpsychological and the intrapsychological in individual development. The relationship of social identities to social representations is further explored, in particular through the introduction of a notion of positioning. A second theme is the examination of the figurative nucleus of children's representations of gender, in which the metaphor of sexual reproduction provides the central image.
\end{abstract}

\section{The Development of Social Representations of Gender}

A key proposition of genetic theories is the notion that to understand something one needs to know how it is constructed. In considering social representations from this point of view I want to focus on their ontogenensis, that is the process through which children's thinking, acting and feeling come to be structured in terms of the social representations of their community. In short, how the child becomes a competent social actor.

The child, of course, is born into a world already structured by social representations. Indeed, in so far as the objectification of social representations constitutes realities, these are the realities of the human world into which the child is born. Yet, the circulation of representations around the child does not lead to them being either simply impressed upon the child, or simply appropriated by the child. Rather, their acquisition is the outcome of a process of development, and a focus on this process can in turn illustrate something about the structure of social representations themselves.

In my own work with Barbara Lloyd (Lloyd and Duveen, 1992) I have been concerned with the development of social representations of gender, and I shall take this as my example. In doing so I am limiting the generality of the argument in an important way. For social representations of gender carry with them an imperative obligation that individuals construct a corresponding social identity (Duveen and Lloyd, 1990). We must all develop gender identities as

This paper was presented at the First International Conference on Social Representations, Villa Rufolo, Ravello, Italy, October 1992, and previously printed in Papers on Social Representations, 2(3), 1993.

Correspondence concering this article should be addressed to Dr. Gerard Duveen, Faculty of Social and Political Sciences, University of Cambridge, Free School Lane, Cambridge CB2 3RQ United Kingdom. 
we grow up if we are to become competent social actors-this does not mean, as we shall see, that we all need to construct the same identities, only that there is an obligation to construct an identity. In this gender is distinct from other social representations, where the identity structure is not imperative, but contractual. There is no imperative obliging us to become psychoanalysts, for example, but if we choose to do so then we must contract into a particular representational field (from this point of view the disputes occasioned by Jeffrey Masson's writings can be viewed as contractual disputes).

A particular identity structure is, therefore, a necessary element in social representations. My focus on gender is limited, therefore, to representations which carry an imperative obligation to identity formation. The development of social representations with different identity structures may follow different paths. In the rest of this paper I shall be drawing on our recent research which investigated the development of gender identities through the first year of schooling. We studied about 100 children in four different schools, following them through their first year (which in England means that they had their 5th birthday during the course of the year). The investigations included ethnographic observations, structured observations, and interview measures.

\section{The Development of Gendered Identities}

An identity can be considered as a psychological process through which meanings are organised and which enables the person to position themselves as a social actor. Social identity in this sense is a way of organising experience which contributes towards the definition of self, but does so by locating the self within the collective world. We have spoken about the expression of social identities since it is through the activities and practices of children that we have access to the social identities which organise such activities. In the course of constructing an identity children draw upon the social representations available to them and in doing so also locate themselves in a particular position within this collective of meaning. For the young child meanings are more clearly established through their practical activity than their intellectual understanding. As our research has illustrated, the social marking of people (themselves and others), of material culture and of space provides the scaffolding which enables children to sustain an organised gender identity. When children were interviewed about their understanding, the support of this collective practice was no longer available to them, and their responses were noticeably less clearly organised.

This sequence in the development of social representations running from practice to reflective knowledge calls to mind the Vygotskyian formula that development proceeds from the interpersonal to the intrapersonal:

'Every function in the child's cultural development appears twice: first on the social level, and later, on the individual level; first between people (interpsychological), and then inside the child (intrapsychological)'. (Vygotsky, 1978, p.57).

Moscovici (1990) voices some doubts about this notion of internalisation, suggesting that it appears too glib, 'too good to be true' (p.179) as he puts it. What disturbs Moscovici is that Vygotsky's formula suggests a direct relationship between social practices and individual functioning. In Vygotsky's work there is no reference to any structures or processes mediating between the intrapsychological and the intrepsychological, yet, as our research has indicated, something does mediate between these two planes of development. For example, data from an interview measure of children's toy preferences suggested that while children used the category of gender to organise their choice of toys, they mistook their own idiosyncratic beliefs about the way in which particular items were marked for gender for consensual beliefs. This result does 
not fit with a notion of development as the simple passage from the interindividual to the intraindividual. If that were the case one might have expected children to have selected as their preference toys which were clearly marked in collective practices in the classroom.

A second difficulty with the Vygotskyian formula is that the same set of collective practices gives rise to differentiated gender identities. Our observations suggested that there is little correspondence between our measure of gender identity in the Autumn Term and in the Summer Term. Across the first year of schooling there is only limited consistency in children's social practices. It is not possible to predict the frequency with which children associate in single sex groups at the end of the school year from a knowledge of how they behaved in the Autumn Term. Yet, even in the Autumn Term children responded to the gender marking of various aspects of material culture and space. In other words, the social practices in each classroom had established clear systems of gender marking, even in at the beginning of the school year. What changed through the year was the way in which individual children positioned themselves in relation to this collective system of meaning. This process was clearer among the girls than among the boys, but it remains unclear how this process of positioning can be accounted for by the Vygotskyian formula.

What is missing from Vygotsky's account is an appreciation of the significance of social identities as the structures mediating between the interpsychological and the intrapsychological. His formula describes a world in which every individual acquires the same understanding from a single set of social practices. Yet where differences emerge between individuals in relation to the same system of collective meanings this formula is inadequate. It may be worth pointing out that Vygotsky's own researches did not extend into areas of social life where differences between individuals become significant. For a Marxist psychologist, Vygotsky shows a curious indifference to the importance of social groups. However suggestive his insistence on the developmental significance of social practices may be, his work does not offer a ready made social psychology.

In our account of the development of social representations of gender we have emphasised the importance of social identities as the structures differentiating between groupings of individuals. Within sex groups, social representations of gender offer a variety of possible gender identities, enabling individuals to position themselves in a number of different ways. Eachtype of social identity provides a certain version of femininity or masculinity, and for the child, different types of social gender identity provide both a means for orienting themselves in the social world of the classroom and a pathway towards the development of their gender identity in later years. For the positions which children adopt also structure their experiences in the classroom and beyond.

In one of the classrooms we observed, for example, some girls had elaborated a feminine identity which excluded boys while others adopted a position in which their femininity could also encompass relations with boys. These two groups of girls are finding different ways of being female, and one consequence of this is a differentiation in their practical experiences in the classroom. Why individual children come to position themselves in different ways may be an interesting question, but it is not one which our research was designed to address. What we can see from our research is that the solutions which children find to the problem of how to position themselves follow patterns which are established within the representational space of gender. In other words, social representations of gender also mark out positions which offer viable gender identities. Viability refers to the extent to which positions within the gender system are consistent with the norms and values of the system. An extreme example can help to clarify this point. It 
is possible for a girl to engage consistently with boys in masculine marked activities. The position of 'tomboy' is a viable identity for a girl. Yet the reverse, a boy who consistently engages with girls in feminine activities, is an untenable position. A boy who did this would become the focus for concern on the part of teachers and parents.

The development of social gender identities refers, then, not to the way in which children negotiate an individual resolution to the question of situating themselves in the world of gender, but, rather, to their adoption of positions which are more or less clearly marked within the social representations of gender. Positioning in this sense has rarely been examined in work on social representations, which have, for the most part, studied the emergence of divergent representations in different groups. The process through which the same representation gives rise to different social identities has been less frequently studied. An exception has been the work on representations of intelligence by Mugny and Carugati (1989), and the related work on mother's representations of children by Molinari and Emiliani (1990). In these studies the different social identities which emerged were related systematically to the social positions occupied by different people. Mugny and Carugati, for example, describe the way in which the theme of intelligence appears from the position of parents or teachers, and others who are more removed from daily contact with children. They suggest that it is parents and teachers need to generate an acceptable explanation for differences between children which accounts for their adherence to an ideology of giftedness. In this account the process of positioning refers to the social-psychological consequences of adopting a particular social role. Our own work has a slightly different focus, and not only because it deals with gender rather than intelligence. Our concern has been with the emergence of children with gendered identities in the context of entering full time schooling.
The process of positioning here not only refers to the way in which children locate themselves within representational space, but it is also a process which, as we have seen, takes place in time.

The theme of time is, in fact, multi-layered in the development of social gender identities. One issue which our research raises but does not answer is the consequences of adopting a particular position within the social world of gender. As we have seen, gender identities defined through our observational measure varied considerably across the first year of schooling. Some positions, though, may offer a more restricted possibility for change than others. Girls who adopt the position of a femininity organised around the exclusion of boys will find very little opportunity for interacting with boys, especially in peer organised contexts. The positions which are adopted do have consequences for the further elaboration of social gender identities. In this sense, social identities not only orient children to the world of the present, but also to the future. From our own work it is difficult to see how clearly this future orientation is apparent to the children themselves. The teachers (and parents) clearly do have representations of how children develop, and these representations are also linked to gender. Different expectations arise of the how girls and boys will develop. To return to the tomboy and the sissy, these are roles which have a clearly specified career path into the future. The tomboy is tolerated because there is an expectation that sooner or later puberty will intervene to reorient the girl into an identity which will enable her to take an appropriate position within the adult world of gender. The anxieties aroused by the sissy are precisely focused on what playing with things feminine signifies for the future sexual identity of the boy. Whether or not children are aware of the temporal dimension of their identities, each of theses different positions points the child along the path of a gender career.

What we are suggesting is that the positions 


\section{Gerard Duveen}

occupied by different social gender identities may vary in the extent to which they constrain the child's future development. From this point of view the identity which a child brings into any social interaction will be an important influence on the course of the interaction and how meanings are negotiated through it. This contrasts with social-psychological theories which have drawn on post-structuralist writings to argue that children's 'subjectivity' is "constituted and reconstituted through the various discursive practices in which they participate" (Davies, 1989, p.229). Such theories emphasise the positioning of the self in relation to specific discourses, but they do so in terms which rarely stretch beyond the immediate horizons of a particular interaction. It is as though positions can be endlessly taken up and changed as children move in and out of different interactions. Yet are gender identities as mobile and flexible as this suggests? Is this not too synchronic a view, which excludes the diachronic consequences of taking up a specific position? Our own view is that the constraints of different positions constitute a kind of inertia which both carries the child into some kinds of interactions rather than others, as well as generating a more fixed identity than such discursive positioning allows.

The question of how social gender identities are elaborated over time requires further research. The first year of schooling is only one moment in a more extended developmental process through which social gender identities are constructed. Even by the end of their first year, children have not yet elaborated the gender identities which are characteristic of adolescence or adulthood. In this sense the parameters of our research do not allow us to reflect upon the whole developmental process through which social representations of gender are internalised and gender identities constructed.

\section{Sexuality and Understanding in}

\section{Children's Representations of Gender}

Central to social representations of gender is a reproductive metaphor which offers an image of gender in terms of the bipolar opposition of the masculine and feminine. This is an image which children appear to have acquired very early in their lives and which persists into adulthood (De Rosa, 1987, also notes that the iconic aspects of social representations of madness are acquired early in life). In discussing the process of objectification Moscovici refers to the figurative nucleus of a social representation, "an image structure that reproduces a conceptual structure in a visible manner" (Moscovici, 1981). The most graphic examples of iconic aspects of social representations of gender in our work concern children's evocation of sexuality in their play. Indeed, sexuality is evoked precisely as the union of bipolar opposites, and once established is celebrated through the rituals of marriage and domestic life. Indeed for these children there is a syncretic fusion of sexual relations, the institution of marriage and the complementarity of gender roles in domestic life. The structure of a bipolar opposition is the connecting thread between these different elements, each of which implicates the others, so that when one element is evoked in play it can lead to the evocation of the others.

The figurative nucleus of bipolar opposites also supports a conceptualisation of social life in terms of two complementary but exclusive categories. This conceptual structure influences how children interpret the world around them, while their participation in collective life provides a scaffolding which confers further legitimacy on this conceptual structure. In sexuality, or more precisely heterosexuality, difference is both asserted because it depends on the presence of bipolar opposites, and also overcome at the same time through the union of these opposites. Sexuality, therefore can take on a privileged status for young children because it offers the clearest resolution to the problem of difference. As we argued earlier, the image of bipolar opposition connects sexuality with marriage and domestic life, and in their play children's 
engagement with this theme expresses and celebrates a certain understanding of the world. In this understanding sex and gender are reduced to a single dimension, and it is the difference between the categories of masculine and feminine which are emphasised, while differences within each of these categories are obscured.

Our observations suggested that children are often the most conservative elements in the gender culture of the classroom. The image of bipolar opposition as the figurative nucleus of their social representations of gender suggests why this should be so. As an image it offers a degree of clarity and simplicity which is also consistent with their limited capacity for any cognitive elaborations which requires greater sophistication. Children's resistance to any influence of an egalitarian voice in representations of gender is also a resistance to losing this clear and sharp image of the world.

Thus this image of bipolar opposition crystallises for the child a state of understanding which also fuses the from of knowledge (its categorical structure) with the content of knowledge (the separation between things masculine and things feminine). All things masculine tend to cohere together and to separate from things feminine. As we noted in our ethnography, separation along these lines can come to characterise the pattern of interaction in the classroom, and once established in this way the dynamic interplay of activity and understanding is capable of sustaining such moments over extended periods of time.

Yet as well as representing difference the image of gender as a bipolar opposition also represents hierarchy, for the difference between the genders is also relation of power. As much as the image is saturated with notions of hierarchy and power, so long as the difference between the bipolar opposites can be resolved, the hierarchy can be obscured. The union of bipolar opposites in sexuality, or the complementarity of marriage and domestic roles, presents an image in which the aspect of hierarchy is masked. Yet the masculine and the feminine are not equals, and the shadow which this inequality casts can be observed in the disputes which break out over access to resources, and in the psychological patterns of overvaluing same gender group and devaluing the opposite gender. For the girls, of course, the reproduction of the hierarchy of gender also brings with it the devaluation of their own gender. It is perhaps not surprising that it is among girls that we have seen evidence of a break with the hegemony of a strict bipolar opposition. From this point of view the girls refusal to attribute as feminine any socially undesirable behaviours in one of our interview measures can be seen as a one such indication. In our ethnography we also recorded a number of episodes of girls challenging this image, usually by competing with boys for masculine marked resources. In comparison to the dominance of an image of gender as a bipolar opposition, these examples are marginal to the general run of life in the reception class. Yet they serve to illustrate that even among the children gender is not an entirely uncontested rerrain, even here relations of power can generate resistance.

\section{Conclusions}

In this brief account of some our research results I have emphasised two themes which are important for a consideration of the development of social representations. First, there is considerable diversity in the identities which children elaborate, and this diversity points to the importance of a mediating structure between interpsychological and intrapsychological functioning. Second, I have emphasised the constructive role of children themselves in elaborating representations of gender, and suggested that, at least in part, this reflects the influence of their developing psychological capacities. This raises the ghost of Piaget, but it also raises the question of which Piaget, for on these issues in particular he wrote in many voices. A constructive engagement with the classical theories of Piaget and Vygotsky may also contribute to the 


\section{Gerard Duveen}

further elaboration of the theory of social representations.

\section{References}

Davies, B. (1989). The discursive production of the Male/Female dualism in school settings. Oxford Review of Education, 15, 229-41.

de Rosa, A. S. (1987). The social representations of mental illness in children and adults. In W. Doise, \& S. Moscovici (Eds.), Current issues in Social Psychology, Vol 2. Cambridge: Cambridge University Press.

Duveen, G., \& Lloyd, B. (1990). Introduction. In G. Duveen, \& B. Lloyd (Eds.), Social Representations and the Development of Knowledge. Cambridge: Cambridge University Press.

Lloyd, B., \& Duveen, G. (1992). Gender Identities and Education: The impact of starting school. London: Harvester-Wheatsheaf.

Molinari, L., \& Emiliani, F. (1990). What is an image? The structure of mothers' images of the child and their influence on conversational styles. In G. Duveen, \& B. Lloyd (Eds.), Social Representations and the Development of Knowledge. Cambridge: Cambridge University Press.

Moscovici, S. (1981). On social representation. In J. Forgas (Ed.), Social Cognition. London: Academic Press.

Moscovici, S. (1990). Social psychology and developmental psychology: Extending the conversation. In G. Duveen, \& B. Lloyd (Eds.), Social Representations and the Development of Knowledge. Cambridge: Cambridge University Press.

Mugny, G., \& Carugati, F. (1989). Social Representations of Intelligence. Cambridge: Cambridge University Press.

Vygotsky, L. (1978). Mind in Society. Cambridge, Mass: Harvard University Press.

(Received Dec. 24, 1995; Accepted Jan. 31, 1996) 